\title{
Application of Single Incision Laparoscopic Surgery for Appendectomies in Patients with Complicated Appendicitis
}

\author{
Kyung Chae Kang, Seok Youn Lee, Dong Baek Kang, Seung Ho Kim, Jung Taek Oh, Duk Hwa Choi ${ }^{1}$, \\ Won Cheol Park, Jeong Kyun Lee \\ Departments of Surgery and ${ }^{1}$ Anesthesiology, Digestive Disease Research Institute and Institute of Medical Science, Wonkwang University \\ College of Medicine, Iksan, Korea
}

Purpose: Recently, single incision laparoscopic surgery (SILS) has been studied for its being less invasive surgery and having cosmetic improvement. We investigated the application of SILS for an appendectomy (SILS-A) in cases of complicated appendicitis and compare it with a conventional laparoscopic appendectomy (C-LA).

Methods: This study involved a total of 40 patients who underwent C-LA or SILS-A in patients with complicated appendicitis; 25 patients received a C-LA, and the other 15 patients received a SILS-A. The clinical outcomes and cosmetic results were compared between the groups.

Results: The SILS-A procedures were performed successfully in patients with complicated appendicitis, but 6 patients who underwent SILS-A needed an additional port for dissection and drainage. Clinical outcomes and postoperative complications were similar in both study groups. The SILS-A group showed significantly higher numbers of pain control than the C-LA group, and the one port SLLS-A group showed significantly better cosmetic result than the C-LA group.

Conclusion: SILS-A is technically feasible and safe in patients with complicated appendicitis. However, SILS-A has more postoperative pain than C-LA, and more active pain control should be considered for patients undergoing SILS-A.

Keywords: Single incision; Laparoscopy; Appendicitis

\section{INTRODUCTION}

Acute appendicitis is the most common disease requiring emergency surgery in the surgical field, and the appendectomy is one of the most basic surgeries for surgeons. In numerous studies, when the conventional laparoscopic appendectomy using 3 ports (C-LA) is compared with a laparotomy, it has advantages of reduced pain, reduced hospital stay, and enhanced esthetic effects [1-3]. Recently, as minimal invasive surgeries draw attention, laparoscopic surgery is being applied to almost all surgical areas, and single incision laparoscopic surgery (SILS)

Received: August 12, 2010 Accepted: October 6, 2010

Correspondence to: Jeong Kyun Lee, M.D.

Department of Surgery and Institute of Medical Science, Wonkwang

University College of Medicine, 344-2 Sinyoung-dong, Iksan 570-711, Korea

Tel: +82-63-859-1492, Fax: +82-63-855-2386

E-mail: rjk@wonkwang.ac.kr

(c) 2010 The Korean Society of Coloproctology

This is an open-access article distributed under the terms of the Creative Commons Attribution NonCommercial License (http://creativecommons.org/licenses/by-nc/3.0) which permits unrestricted non-

commercial use, distribution, and reproduction in any medium, provided the original work is properly cited. is being applied to diverse surgeries as a new technique for minimal invasive surgery [4-7].

In studies comparing single incision laparoscopic surgery for an appendectomy (SILS-A) with a conventional laparoscopic appendectomy, although early pain was observed, the former was superior from the esthetic viewpoint, and the incidence of complications was not different. Thus, recently, it was reported as a technique that could be performed safely [8-10]. However, studies on the application of single incision laparoscopic surgery for an appendectomy to cases of complicated appendicitis associated with necrosis, perforation, or abscess have not been reported. Therefore, we performed this study to examine the application and the safety of SILS-A by comparing single incision laparoscopic surgery for an appendectomy with a conventional 3-port laparoscopic appendectomy.

\section{METHODS}

\section{Subjects}

The study was performed on 40 appendicitis patients with complications who underwent either a 3-port laparoscopic 
appendectomy or single incision laparoscopic surgery for an appendectomy by the same surgeon from July 2009 to June 2010 at our hospital. Patients receiving a 3-port C-LA were 25 cases, and patients receiving single incision laparoscopic surgery for an appendectomy were 15 cases. Among the patients who underwent single incision laparoscopic surgery for an appendectomy and the patients who underwent a 2-port laparoscopic appendectomy, due to severe inflammation and severe adhesion, resection was difficult; thus, additional trocars were inserted for drainage in 6 cases. Patients with other diseases, such as previous cardiopulmonary diseases, uncontrollable diabetes, and renal failure, and thus a risk group for general anesthesia and the pneumoperitoneum space, and 2 patients converted to a laparotomy due to adhesion were excluded from the subjects. This study was a retrospective review of medical records. The technique of single incision laparoscopic surgery for an appendectomy was approved by the Ethical Committee of our hospital.

Prior to surgery, abdominal ultrasonography or computed tomography was performed on all patients. In regard to surgical methods, the 3-port laparoscopic appendectomy, single incision laparoscopic surgery for an appendectomy and the laparotomy were explained to the patient, after which the method was selected by the patients themselves and their guardians. While explaining laparoscopic surgery, the conversion to a was explained again. Appendicitis associated with complications are defined as cases showing gangrene or perforation changes detected by surgical findings or histologically, or cases with an abscess in the vicinity of the appendix.

\section{Surgical methods}

General anesthesia was administered to all patients, drainage was performed prior to surgery, and the insertion of a drainage tube was performed on two patients with generalized peritonitis. Simultaneously with the diagnosis of appendicitis, an antibiotic, 2nd generation cephalosporin, was administered, and for cases diagnosed as appendicitis associated with complications, aminoglycoside and metronidazole were also administered.

In the supine position, the patient took an approximately 15 $20^{\circ}$ trendelenburg position, and the surgical view was secured by leaning to the left by $15-20^{\circ}$. The surgeon stood in the left lower area of the patient, leaning toward the lower extremities, and the first assistant manipulated the laparoscope on the right upper side of the surgeon. The 3-port laparoscopic appendectomy was performed using 3-trocar techniques, and through the transverse incision of the skin, $1.5-2 \mathrm{~cm}$ in length, in the vicinity of the umbilicus, the pneumoperitoneum was made using a Veress needle, and a 10-mm trocar was inserted. A 5$\mathrm{mm}$ trocar was inserted between the pubic bone and the middle of the umbilicus, and another 5-mm trocar was inserted in the vicinity of the McBurney point. The mesoappendix was li- gated and dissected by the application of the LigaSure (Valleylab, Boulder, CO, USA) and electric coagulation. The appendiceal base was ligated by the use of one Endo loop (Ethicon, Somerville, NJ, USA), and electric coagulation was performed on the mucosa of the dissected appendix. To prevent infection in the area of the trocar insertion, the surgeon removed the resected appendix to the extracorporeal area by using a Lap-bag (Sejong Medical, Paju, Korea) through the 10-mm trocar area. The abdominal cavity was washed with saline. Afterward, for cases showing perforation or severe inflammation, such as an abscess in the vicinity of the appendix, sufficient drainage after surgery was achieved by installing a Jackson-pratt drain through the 3rd trocar.

Single incision laparoscopic surgery for an appendectomy was performed in the supine position under general anesthesia, and in the umbilical area, according to the open incision method, a $1.5-$ to $2-\mathrm{cm}$ vertical incision was made. If the umbilical area was severely dirty or malodorous, avoiding the center of the umbilical area, in the area above the umbilical area or based on the umbilical area, a half moon incision window $1.5-$ to $2-\mathrm{cm}$ in size was made. When the insertion route to the abdominal cavity was secured, a wound retractor (Alexis, Applied Medical, Rancho Santa Margarita, CA, USA) was inserted, $30^{\circ}$. In addition, one $5-\mathrm{mm}$ trocar for use with a $30^{\circ}, 5-\mathrm{mm}$ laparoscopic camera (Karl-Storz, Tuttlingen, Germany) and the injection of $\mathrm{CO}_{2}$ gas, two homemade 5-mm trocars to reduce collisions of the tips of the trocars during single incision laparoscopic surgery, and a three-way catheter to remove smoke generated during the use of the electric coagulator were fixed using silk in the finger area of surgical gloves to prevent the leakage of the air (Fig. 1A). To prepared the homemade 5-mm trocars, the wide upper area of the previous 5-mm trocar was eliminated, and an elastic rubber hose was connected to the end of the column. If the elastic rubber hose were to be located above, during the manipulation of the laparoscopic device, the trocar would be readily depressed to the inside of the glove; thus, it was located below and immobilized in the finger area inside the surgical gloves (Fig. 1B). For cases difficult to resect because of perforation or severe inflammation, such as an abscess in the vicinity of the appendix and requiring drainage, an additional 5$\mathrm{mm}$ trocar was inserted in the vicinity of the McBurney point. The intra-abdominal cavity pressure was maintained by using a method similar to that used for conventional laparoscopic surgery, and a meso-appendectomy and an appendectomy were performed by using identical methods. After the resection of the appendix, the resected appendix was added to the finger of glove that was no longer required and ligated with a pair of forceps. If the appendix was big or contamination was severe, using the Lap-bag, it was removed to the extracorporeal area, the abdominal cavity was washed, and the wound retractor was removed. If drainage was required, a 5-mm trocar was inserted in the vicinity of the McBurney point, and a Jackson-pratt drain 
was installed.

\section{Postsurgical pain management}

In all patients, a patient-controlled analgesia (Accufusor, WooYoung Medical, Korea) was used. As the patient-controlled analgesia, $18 \mu \mathrm{g} / \mathrm{kg}$ of fentanyl and $3 \mathrm{mg} / \mathrm{kg}$ of Keromin (Ketorolac Tromethamine) were diluted with metoclopramide and saline to a $100-\mathrm{mL}$ volume and injected. For cases presenting with severe pain, higher than 5 points on a verbal numerical rating scale (VNRS), despite the use of patient-controlled analgesia, as additional analgesic, Keromin (Ketorolac Tromethamine) was injected intravenously.

\section{Data analysis}

The subject patients were divided into the group that underwent a 3-port laparoscopic appendectomy and the group that underwent single incision laparoscopic surgery for an appendectomy. The group who underwent single incision laparoscopic surgery for an appendectomy was divided again into the group that underwent one-port single incision laparoscopic surgery for an appendectomy and the group that underwent two-port single incision laparoscopic surgery for an appendectomy, and operation time (duration from skin incision to skin suture), the postsurgical hospitalization period, the gas release time after surgery, the day oral intake was initiated, complications, the frequency of the administration of analgesics after surgery, and the esthetic satisfaction level were analyzed by using the student's t-test and the chi-square test with the SPSS program (SPSS Inc., Chicago, IL, USA). P-values lower than 0.05 were considered to be statistically significant. Esthetic satisfaction level was scored by interviews, with 0 being unsatisfactory to 10 being satisfactory, at the first visit to an outpatient clinic after discharge.

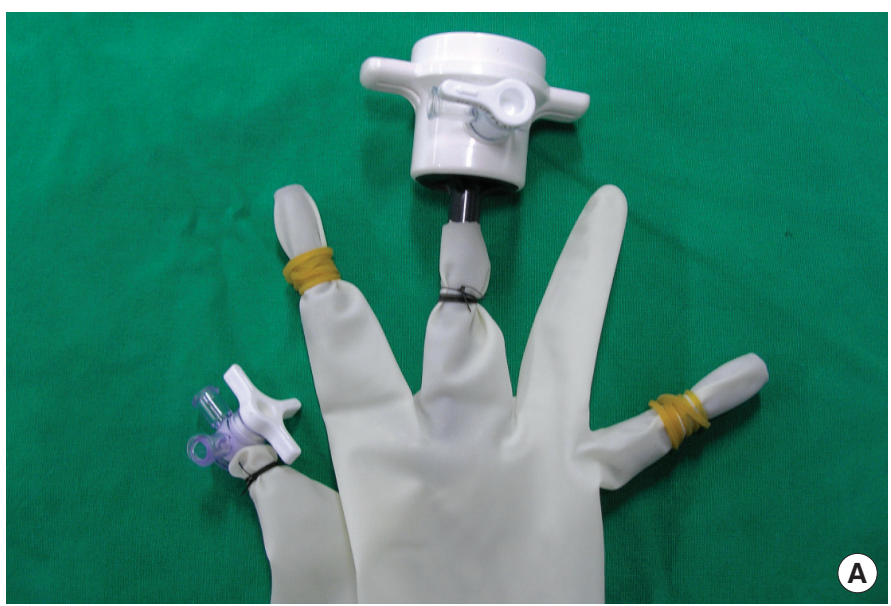

\section{RESULTS}

In patients who underwent single incision laparoscopic surgery for an appendectomy, cases in which the resection was difficult due to severe inflammation resulting in severe adhesion and additional trocars had to be inserted for drainage were 6 , but none of those cases was converted to 3-port laparoscopic appendectomy or a laparotomy. The ratio of males to females for the patients who underwent single incision laparoscopic surgery for an appendectomy was 8:7, their mean age was 35.5 \pm 13.2 years, and their body mass index was an average $23.4 \pm$ $3.2 \mathrm{~kg} / \mathrm{m}^{2}$. In the group that underwent a 3-port laparoscopic appendectomy, the ratio of males to females was 14:11, their age was $37.9 \pm 14.5$ years, and their body mass index was 24.2 $\pm 3.6 \mathrm{~kg} / \mathrm{m}^{2}$. The operation time of the group that underwent single incision laparoscopic surgery for an appendectomy was $62.5 \pm 18.7$ minutes, that of the group that underwent single

Table 1. Demographic data and operative comparison for C-LA and SILS-A in complicated appendicitis

\begin{tabular}{lccc}
\hline & C-LA $(n=25)$ & SILS-A $(n=15)$ & P-value \\
\hline Gender (M/F) & $14 / 11$ & $8 / 7$ & 0.752 \\
Mean age \pm SD (yr) & $37.9 \pm 14.5$ & $35.5 \pm 13.2$ & 0.624 \\
Mean BMI \pm SD & $24.2 \pm 3.6$ & $23.4 \pm 3.2$ & 0.867 \\
Mean OP time (min) & $53.7 \pm 11.5$ & $62.5 \pm 18.7$ & 0.084 \\
Time until gas out (hr) & $29.5 \pm 14.7$ & $35.2 \pm 18.5$ & 0.134 \\
Time until diet start (hr) & $45.2 \pm 12.6$ & $50.3 \pm 14.5$ & 0.231 \\
Hospital stay (day) & $6.4 \pm 1.6$ & $6.8 \pm 1.8$ & 0.840 \\
No. of IV pain control & $0.7 \pm 0.6$ & $2.2 \pm 1.5$ & 0.045 \\
Cosmetic results & $8.5 \pm 1.5$ & $9.2 \pm 0.8$ & 0.086 \\
\hline
\end{tabular}

C-LA, three-port conventional laparoscopic appendectomy; SILS-A, single incision laparoscopic surgery for appendectomy; SD, standard deviation.

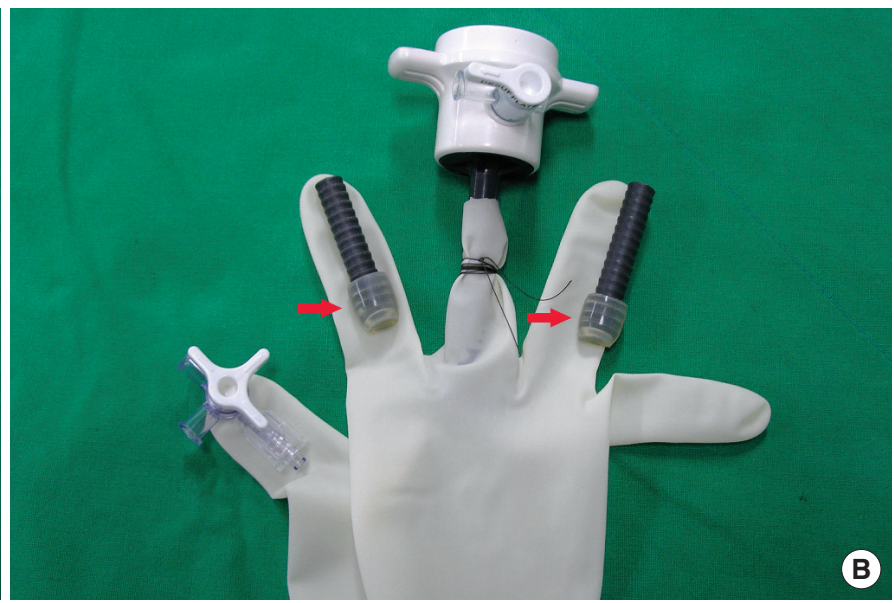

Fig. 1. Homemade port system of single incision laparoscopic surgery for appendectomy. A surgical glove was attached with a 5-mm trocar, two pipes and one 3-way (A), and an elastic rubber hose (arrow) was attached at the distal pipe (B). 
port single incision laparoscopic surgery for an appendectomy was $60.5 \pm 15.8$ minutes, and that of the group that underwent a 3-port laparoscopic appendectomy was $53.7 \pm 11.5$ minutes. Although the time was longer for the group that underwent single incision laparoscopic surgery for an appendectomy, no statistically significant differences was detected $(P=0.084,0.173)$.

As clinical courses, the time to the first gas out after surgery of the group that underwent a single incision laparoscopic appendectomy was $35.2 \pm 18.5$ hours, that of the group that underwent one-port single incision laparoscopic surgery for an appendectomy was $31.3 \pm 15.0$ hours, and that of the group that underwent a 3-port laparoscopic appendectomy was $29.5 \pm 14.7$ hours. The times to the first diet after surgery were $50.3 \pm 14.5$ hours, $47.4 \pm 11.6$ hours, and $45.2 \pm 12.6$ hours, and the hospitalization periods were $6.8 \pm 1.8$ days, $6.2 \pm 1.5$ days, and $6.4 \pm$ 1.5 days, respectively. The interval to the first gas out after surgery, the start of diet, and the hospitalization period showed no statistically significant difference.

The frequency of additional analgesics administered to the single incision laparoscopic surgery for an appendectomy group was $2.2 \pm 1.5$ times, that for the one-port single incision laparoscopic surgery for an appendectomy group was $1.3 \pm 1.8$ times, and that for the 3-port laparoscopic appendectomy group was $0.7 \pm 0.6$ times. The frequency in the single incision laparoscopic surgery for an appendectomy group, in comparison with that for the 3-port laparoscopic appendectomy group $(\mathrm{P}=0.045)$ was higher, and this result had statistical significance, but the comparison with the one-port single incision laparoscopic appendectomy group showed no statistically significant difference $(\mathrm{P}=0.088)$.

The esthetic satisfaction levels for the single incision laparoscopic surgery for an appendectomy group and for the 3-port laparoscopic appendectomy group were $9.2 \pm 0.8$ points and $8.5 \pm 1.5$ points, respectively, but this difference was not statistically significant. Nonetheless, the one-port single incision laparoscopic surgery for an appendectomy group showed the statistically significant improved cosmetic results $)(P=0.043)$ (Tables 1, 2).

In regard to postsurgical complications, in the single incision laparoscopic surgery for an appendectomy group, seroma in the umbilical area developed in 2 patients, wound infection in the umbilical area developed in 1 patient, and a defect of the fascia in the umbilical area developed in 1 patient, significant these differences between the two groups were not statistically significant (Tables 3, 4). When the defect of the fascia in the umbilical area and seroma developed simultaneously, the fascia defect was sutured 7 days after surgery. The other patients recovered after preservation treatments performed at outpatient clinics. In the 3-port laparoscopic appendectomy group, wound infection developed in 1 patient, ileus developed in 1 patient; they recovered after conservative management. Fig. 2 is immediated postoperative scar after SILS-A.

\section{DISCUSSION}

Since the laparoscopic appendectomy was introduced for the first time by Semm [11] in Germany for an appendix without inflammation during gynecological surgery using laparoscopy, it has been performed by numerous surgeons. In comparison with a laparotomy, a laparoscopic appendectomy reduces post-

Table 3. Postoperative complications in C-LA and SILS-A

\begin{tabular}{lccc}
\hline & C-LA $(n=25)$ & SILS-A $(n=15)$ & P-value \\
\hline Wound seroma & 0 & 2 & 0.164 \\
Wound infection & 1 & 1 & 0.717 \\
Ileus & 1 & 0 & 0.327 \\
Fascia dehiscence & 0 & 1 & 0.334 \\
Overall (\%) & $2(8)$ & $3(20)^{\mathrm{a}}$ & 0.330 \\
\hline
\end{tabular}

C-LA, three-port conventional laparoscopic appendectomy; SILS-A, single incision laparoscopic surgery for appendectomy.

${ }^{a}$ Wound seroma and fascia dehiscence were present in the same patient.

Table 2. Demographic data and operative comparison for C-LA, Tp SILS-A, and Op SILS-A in complicated appendicitis

\begin{tabular}{|c|c|c|c|c|c|}
\hline & C-LA $(n=25)$ & Tp SILS-A $(n=6)$ & P-value & Op SILS-A $(n=9)$ & P-value \\
\hline Gender (M/F) & $14 / 11$ & $4 / 2$ & 0.451 & $4 / 5$ & 0.824 \\
\hline Mean age \pm SD (yr) & $37.9 \pm 14.5$ & $34.2 \pm 16.7$ & 0.357 & $38.2 \pm 11.7$ & 0.767 \\
\hline Mean OP time (min) & $53.7 \pm 11.5$ & $64.8 \pm 23.5$ & 0.078 & $60.5 \pm 15.8$ & 0.173 \\
\hline Time until gas out (hr) & $29.5 \pm 14.7$ & $36.9 \pm 21.5$ & 0.154 & $32.6 \pm 15.0$ & 0.551 \\
\hline Hospital stay (day) & $6.4 \pm 1.6$ & $7.2 \pm 2.8$ & 0.240 & $6.2 \pm 1.5$ & 0.825 \\
\hline No. of IV pain control & $0.7 \pm 0.6$ & $2.6 \pm 2.0$ & 0.040 & $1.6 \pm 1.5$ & 0.088 \\
\hline Cosmetic results & $8.5 \pm 1.5$ & $9.0 \pm 1.0$ & 0.245 & $9.5 \pm 0.5$ & 0.043 \\
\hline
\end{tabular}

C-LA, three-port conventional laparoscopic appendectomy; Tp SILS-A, two-port single incision laparoscopic surgery for appendectomy; Op SILS-A, one-port single incision laparoscopic surgery for appendectomy; SD, standard deviation. 
Table 4. Postoperative complications in C-LA, Tp SILS-A, and Op SILS-A

\begin{tabular}{lccccc}
\hline & C-LA $(\mathrm{n}=25)$ & Tp SILS-A $(\mathrm{n}=6)$ & P-value & Op SILS-A $(\mathrm{n}=9)$ & 1 \\
\hline Wound seroma & 0 & 1 & 0.363 & 0 & 0.096 \\
Wound infection & 1 & 1 & 0.490 & 0 & 0.55 \\
lleus & 1 & 0 & 0.327 & 0 & 0.55 \\
Fascia dehiscence & 0 & 1 & 0.363 & $1(11.1)$ & 1.0 \\
Overall (\%) & $2(8)$ & $2(30)^{\mathrm{a}}$ & 0.291 & 0.786 \\
\hline
\end{tabular}

C-LA, three-port conventional laparoscopic appendectomy; Tp SILS-A, two-port single incision laparoscopic surgery for appendectomy; Op SILS-A, one-port single incision laparoscopic surgery for appendectomy.

${ }^{a}$ Wound seroma and fascia dehiscence were present in the same patient.
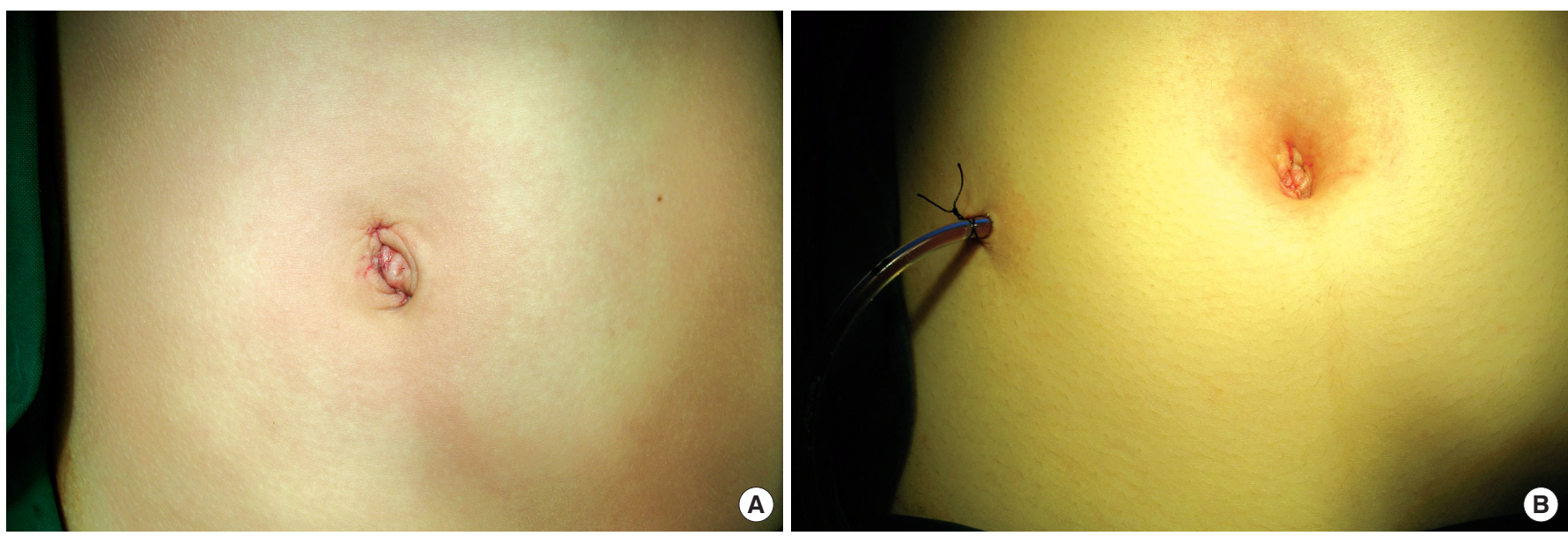

Fig. 2. Immediate postoperative scar after single incision laparoscopic surgery for appendectomy. An 18-year-old, gangrenous-type woman patient (A), and a 25-year-old, perforated-type woman patient (B).

surgical pain and the dose of analgesics. It also reduces not only tissue injury of patients but also irritation of the intestine and, thus, reduces adhesion that may occur after surgery. It allows early ambulation and food intake, it shortens the hospitalization period, which allows early return to routine life, it allows other associated diseases within the abdominal cavity to be assessed, and it has cosmetic improvements. As a result, it is now widely performed [1-3].

As laparoscopic minimal invasive surgery draws attention, interest in non-scar surgical methods is on the rise, and together with the development of equipment, Natural Orifice Transluminal Endoscopic Surgery (NOTES), single-trocar or singleincision surgical methods have been applied to diverse diseases in the abdominal cavity $[4-7,12]$ Although it differs slightly depending on the surgeon, single incision laparoscopic surgery for an appendectomy makes an incision window through the umbilicus in most cases. It is applied to an appendectomy as a new technique of minimal invasive surgery because the umbilicus is located in the middle of the abdomen, so diverse intraabdominal approaches can be performed; blood vessels and nerves are absent, so incision windows can be readily created; even after surgery, wounds become depressed within the um- bilicus and, thus, may be considered as a congenital existing scar $[8-10,13]$.

Reviewing the reports that compared single incision laparoscopic surgery for an appendectomy with a conventional 3-port laparoscopic appendectomy, the former was found to reduce scars, in addition to having the advantages of a 3-port laparoscopic appendectomy; thus, it is advantageous from cosmetic improvement. Nonetheless, shortcomings, long operation time and substantial early postsurgical pain, have been reported [810]. The reason that the operation time of single incision laparoscopic surgery for an appendectomy is longer than that for a conventional 3-port laparoscopic appendectomy may be that different from the conventional 3-port laparoscopic appendectomy, the location and the view of the laparoscopic camera are limited and a narrow incision window causes collisions with and interference between the laparoscopic surgical equipment and the laparoscopic camera $[14,15]$. To compensate for such shortcomings, we used a $30^{\circ}, 5-\mathrm{mm}$ laparoscopic camera (KarlStorz). If commercial trocars are used during single incision laparoscopic surgery, during the manipulation of laparoscopy, collisions in the wide upper area; thus, to reduce the number of collisions, the wide upper area of the conventional 5-mm was 
eliminated, and only the columnar lower area was made as a homemade movement pathway of the laparoscopic equipment. To prevent the leakage of intra-abdominal gas to the homemade columnar path, an elastic rubber hose was connected to the tip area. When the elastic rubber hose was placed in the upper area, during the manipulation of laparoscopic equipment, the columnar trocar became readily depressed inside the gloves; hence, it was placed in the lower area, fixed in the finger area inside the glove, and laparoscopic manipulation was performed (Fig. 1).

For cases in which collision and interference phenomenon between laparoscopic surgical equipment and laparoscopic cameras occur, laparoscopic manipulation was made easy by using the flexible laparoscopic Roticulator Grasper, Dissector, and Shear (Covidien, Norwalk, CT, USA). If it was determined that resection was difficult because of severe adhesion due to inflammation or that drainage was required, an additional 5$\mathrm{mm}$ trocar was inserted immediately in the vicinity of the McBurney point to facilitate the manipulation of laparoscopy.

Through such methods as those discussed above, single incision laparoscopic surgery for an appendectomy can even be applied to appendicitis patients with associated complications, and the operation time may be shortened. In our study, in appendicitis patients with associated complications, the surgery time was longer than that for a conventional 3-port laparoscopic appendectomy, but the difference was not statistically significant.

Recently, commercial single trocars have been used. Nonetheless, to lower the cost and to convert commercialized trocars to working windows, a minimum $2.5-\mathrm{cm}$ incision window was required. Hence, to remove small organs such as the appendix, large incisions were thought not to be required, so they were not used. For the removal of big organs, the use of commercial single trocars is thought to be convenient, and the operation time may be shortened.

Park et al. [9] reported that in single incision laparoscopic surgery for an appendectomy, early pain was more severe than it was in a conventional 3-port laparoscopic appendectomy. This might be caused by the fact that although the skin incision in the umbilical area is small, the actual length of the fascia incision is longer, and through a small incision window, laparoscopic equipment is used at once, which irritates the incision window. In our study, similarly, in the single incision laparoscopic surgery for an appendectomy group, statistically- significantly more analgesics were administered, and this is thought to be associated with the fascia incision length being longer than the skin incision in the umbilical area, as suggested by Kim et al. [16].

In addition, in the one-port single incision laparoscopic surgery for an appendectomy group, pain was not statistically significantly different; nonetheless, the 2-port single incision laparoscopic surgery for an appendectomy group showed statistically significant differences; thus, pain patterns different from those for one-port single incision laparoscopic surgery for an appendectomy were shown. This may be due to different pain patterns being shown according to not only the length of the fascia incision in the umbilical area but also additionally inserted trocars and clinical features. For such cases, aggressive management of early pain may be required.

Postsurgical complications in patients who underwent single incision laparoscopic surgery for an appendectomy were treated without special side effects or complications, except wound problems. A seroma in the umbilical area developed in 2 patients, wound infection in the umbilical area developed in 1 patient, and a fascia defect in the umbilical area developed in 1 patient. However, these results were not statistically different from those for patients who underwent a 3-port laparoscopic appendectomy. The fascia defect in the umbilical area and the seroma developed simultaneously, and 7 days after surgery, the fascia defect was sutured; nonetheless, the incidence of complications, compared with patients who underwent a 3-port laparoscopic appendectomy, was not statistically different. The other 2 patients recovered after preservation treatment administered in outpatient clinics. All postsurgical complications developed in patients who had had the center of the umbilical area resected vertically. It is thought that, depending on the state of the umbilical area of each patient, if the umbilical area is severely dirty or malodorous, a half-moon incision in the upper umbilical area or near the center of the umbilical area, but avoiding the center, should be considered. As introduced by Kim et al., [16] if the peritoneum and the fascia are sutured together, the surgery can be completed without skin suture, and the umbilicus can be compressed with gauzes formed as a ball, the wound can drain well, and wound inflammation can be reduced. In addition, the umbilicus becomes located within the skin, thus improving the cosmetic results. To prevent a defect of the fascia in the umbilical area, during suture, it is required to make sure definitely the umbilical fascia.

The esthetic satisfaction level was not statistically significant in single incision laparoscopic surgery for an appendectomy. However, in patients who underwent one-port single incision laparoscopic surgery for an appendectomy, statistically significant satisfaction levels were shown in comparison with patients who underwent 3-port laparoscopic surgery for an appendectomy. Thus, if problems associated with wounds are resolved, esthetic satisfaction can be maximized.

In six patients who underwent single incision laparoscopic surgery for an appendectomy, resection was difficult due to severe adhesion, and additional trocars were inserted for drainage. In a strict sense, the surgery in these cases was not single incision laparoscopic surgery for an appendectomy; nonetheless, at first, single incision laparoscopic surgery could be applied to appendicitis patients with associated inflammation, and as mentioned above, single incision laparoscopic surgery for an appendectomy is thought to be applicable to all types of 
appendicitis. In other words, in appendicitis with associated complications, during surgery, if the progression of surgery is difficult, additional trocars may be inserted and used conveniently, and through the port, drainage tubes may be installed. Thus, a single incision laparoscopic appendectomy can be applied to appendicitis with associated complications, and it is thought to be a safe technique. In the future, comparative studies on more patients should be conducted. When single incision laparoscopic surgery for an appendectomy was applied to appendicitis patients with associated complications, surgery could be performed without substantial difficulties during the procedure, and the postsurgical satisfaction level of patients was high. In single incision laparoscopic surgery for an appendectomy, more cases presented with early pain in comparison with a 3port appendectomy. Therefore, aggressive pain management may be required. A series of studies on larger patient groups should be conducted if single incision laparoscopic surgery for an appendectomy is to be established as the preferred surgery for appendicitis patients with associated complications.

\section{CONFLICT OF INTEREST}

No potential conflict of interest relevant to this article was reported.

\section{ACKNOWLEDGMENT}

This paper was supported by Wonkwang University 2008.

\section{REFERENCES}

1. Towfigh S, Chen F, Mason R, Katkhouda N, Chan L, Berne T. Laparoscopic appendectomy significantly reduces length of stay for perforated appendicitis. Surg Endosc 2006;20:495-9.

2. Khan MN, Fayyad T, Cecil TD, Moran BJ. Laparoscopic versus open appendectomy: the risk of postoperative infectious complications. JSLS 2007;11:363-7.

3. Golub R, Siddiqui F, Pohl D. Laparoscopic versus open appendectomy: a meta analysis. J Am Coll Surg 1998;186:545-53.
4. White WM, Haber GP, Goel RK, Crouzet S, Stein RJ, Kaouk JH. Single-port urological surgery: single-center experience with the first 100 cases. Urology 2009;74:801-4.

5. Bucher P, Pugin F, Morel P. Single port access laparoscopic right hemicolectomy. Int J Colorectal Dis 2008;23:1013-6.

6. Kuon Lee S, You YK, Park JH, Kim HJ, Lee KK, Kim DG. Singleport transumbilical laparoscopic cholecystectomy: a preliminary study in 37 patients with gallbladder disease. J Laparoendosc Adv Surg Tech A 2009;19:495-9.

7. Oltmann SC, Rivas H, Varela E, Goova MT, Scott DJ. Single-incision laparoscopic surgery: case report of SILS adjustable gastric banding. Surg Obes Relat Dis 2009;5:362-4.

8. Hong TH, Kim HL, Lee YS, Kim JJ, Lee KH, You YK, et al. Transumbilical single-port laparoscopic appendectomy (TUSPLA): scarless intracorporeal appendectomy. J Laparoendosc Adv Surg Tech A 2009;19:75-8.

9. Park JH, Hyun KH, Park CH, Choi SY, Choi WH, Kim DJ, et al. Laparoscopic vs transumbilical single-port laparoscopic appendectomy: results of prospective randomized trial. J Korean Surg Soc 2010;78:213-8.

10. Vidal O, Valentini M, Ginesta C, Marti J, Espert JJ, Benarroch G, et al. Laparoendoscopic single-site surgery appendectomy. Surg Endosc 2010;24:686-91.

11. Semm K. Endoscopic appendectomy. Endoscopy 1983;15:59-64.

12. Horgan S, Cullen JP, Talamini MA, Mintz Y, Ferreres A, Jacobsen GR, et al. Natural orifice surgery: initial clinical experience. Surg Endosc 2009;23:1512-8.

13. Saber AA, Elgamal MH, El-Ghazaly TH, Dewoolkar AV, Akl A. Simple technique for single incision transumbilical laparoscopic appendectomy. Int J Surg 2010;8:128-30.

14. Esposito C. One-trocar appendectomy in pediatric surgery. Surg Endosc 1998;12:177-8.

15. Ishikawa N, Arano Y, Shimizu S, Morishita M, Kawaguchi M, Matsunoki A, et al. Single incision laparoscopic surgery (SILS) using cross hand technique. Minim Invasive Ther Allied Technol 2009;18:322-4.

16. Kim HJ, Lee JI, Lee SC, Kim SH, Lee IK, Lee YS, et al. Single-port laparoscopic appendectomy. J Korean Surg Soc 2010;78:338-42. 